\title{
The Development Trend of Industrial Wireless Positioning Range
}

\author{
Wang Xiaoqiang ${ }^{1, a}$, Rong Shasha ${ }^{1, b, *}$, Cui Fengkui ${ }^{1, c}$, Su Yongxiang ${ }^{1, d}$, Xie Kege ${ }^{2, e}$ \\ ${ }^{1}$ Electromechanical Engineering College, Henan University of Science and Technology, \\ Luoyang, China \\ ${ }^{2}$ Electromechanical Engineering College, Xi`an Jiaotong University, Xi’an, China \\ a wang_xq2002@163.com, ${ }^{\mathrm{b}}$ 214379737@qq.com, ${ }^{\mathrm{c}}$ cuifk2008@163.com, \\ d635618634@qq.com, ${ }^{\mathrm{d}}$ 1061822031@qq.com
}

Keywords: Wireless positioning; Range; The signal characteristic value

\begin{abstract}
With the improvement of ranging precision, rangefinder has been widely used in industry development. In order to better understand the rangefinder and improve its accuracy. First, we compares the three kinds of rangefinder for advantages and disadvantages, introduces the principle and advantage of the wireless positioning range. Second, this paper briefly describes the research status of related literature at the two aspects of domestic and foreign. Third we compared the advantages and disadvantages of several signal characteristic value, and proposed the new ranging method by radio wavelength. Finally, the industrial wireless positioning technology of the future development trend is forecasted. To improve the ranging accuracy has certain theoretical significance and engineering application value, lay a foundation for the development of industrial wireless range
\end{abstract}

\section{Introduction}

With the development of global positioning system, wireless positioning technology has undergone tremendous changes, especially the difference in the technology, the positioning accuracy of a qualitative leap, greatly enhance the positioning accuracy, civil navigation satellite GPS can reach $5 \mathrm{~m}$, while in the military, aviation precision is higher ${ }^{[1 \sim 2]}$. In industrial field, wireless positioning plays a key role, the accuracy of the size of a part determines the life of the whole machine, and even determines the future development of the enterprise. Therefore, the accuracy of wireless location is worthy of further study.

\section{Overview of industrial rangefinder}

Usually the distance measuring method is using a ruler (meter, micrometer), and for high precision equipment, scale error is too large, it is difficult to meet the needs of the society. At present, the market can be simply summarized in the field of industrial and civil level. General industrial grade range of protection level are relatively high, while the protection level of civil rangefinder is relatively low, usually industrial measuring instruments and equipment used are: laser rangefinder ${ }^{[3]}$, ultrasonic range finder ${ }^{[4]}$, infrared range finder ${ }^{[5]}$.

In these three kinds of range finder, laser range finder and ultrasonic rangefinder are more common. The precision of the laser range finder is the highest among the three, but its cost is the highest, and it is necessary to pay attention to the safety of the human body. Not applicable to small 
manufacturing enterprises. Ultrasonic distance meter cost is not high, but the measurement accuracy is relatively low, will bring losses to precision manufacturing enterprises. Infrared range finder, although the cost is low, easy to make, but the measurement accuracy is the lowest, so it does not apply to manufacturing enterprises also. Through the comparison of the above range finder, the advantages and disadvantages of these three kinds of range finder are shown in table 1.

Table 1 Advantages and disadvantages of three kinds of range finder

\begin{tabular}{|c|c|c|}
\hline Type & Advantage & Disadvantages \\
\hline Laser range finder & $\begin{array}{c}\text { High precision } \\
\text { measurement and } \\
\text { orientation }\end{array}$ & High cost, the need for regular maintenance \\
\hline Ultrasonic Range Finder & Moderate price & $\begin{array}{c}\text { Affected by the surrounding environment, } \\
\text { the general measurement distance is shorter, } \\
\text { the measurement accuracy is relatively low }\end{array}$ \\
\hline Infrared range finder & Cheap, easy, safe & $\begin{array}{c}\text { Low precision, near distance, poor } \\
\text { directivity }\end{array}$ \\
\hline
\end{tabular}

In recent years there has been a microwave range finder, the range finder has the advantages of high precision, wide range, strong anti-interference ability, and the cost of production of microwave range finder is relatively low, therefore, it has attracted the attention of researchers. The principle of the microwave range finder is similar to that of radar, which is measure the delay time from the sending signal of the terminal to receiving the reflected signal of the other terminal. In short, is to measure the time about electromagnetic wave return the radar and the target ${ }^{[6]}$, and then calculate the coordinates of the measured node through algorithm that is the three sides of the method ${ }^{[7]}$, and the maximum likelihood estimation method ${ }^{[8]}$.

\section{Research status at home and abroad}

\subsection{Foreign research status}

The international radio technology came into being in the middle and late nineteenth Century, and its theoretical basis is the electromagnetism founded by Maxwell, a British physicist. In 1895, Marconi implemented the first radio communication, and then German physicist Hertz confirmed that the radio wave transmission has all the characteristics of wave transmission. In 1909, Marconi, the inventor of Italy, improved radio technology and invented wireless telegraphy. After the end of World War II, along with the new technology revolution, With the emergence of a large number of advanced technologies, such as satellite and computer, the application of radio in positioning is more common, which greatly promotes the development of wireless positioning technology ${ }^{[9]}$.

Internationally, satellite positioning mainly based on the United States GPS, Russia's GLONASS, the European Galileo. Among them, the GPS positioning system is one of the most widely used, but it also has some shortcomings, for example, in large cities and mountainous areas, due to the impact of high-rise buildings and trees on the signal, resulting in positioning errors.

In foreign countries, most of the research results of the ground wireless location are positioning algorithm, and the positioning system is realized according to the algorithm, the purpose is to improve the positioning accuracy. The NLOS error is one of the main errors which affect the positioning accuracy. At present, many researchers have done a lot of research on it. McGuire ${ }^{\text {[10] }}$ proposed a robust estimation function, to improve for mobile terminal positioning estimation that can obtain better location estimation in the NLOS environment. According to the RSS model, K. Chintalapudi ${ }^{[11]}$ has designed a kind of indoor positioning algorithm without early deployment will 
be able to locate. In the aspect of positioning technology optimization, Dong-Ho Shin ${ }^{[12]}$ studied the statistical characteristics of error about TOA and TDOA. In addition, Forde L C and Howick E F, scientists of the New Zealand standard laboratory, proposed a method that the method can be used to obtain the periodic error and constant for Photoelectric distance measuring instrument ${ }^{[13]}$, the uncertainty budget of the verification device is $0.39 \mathrm{~mm}$, under the $95 \%$ confidence probability.

\subsection{Domestic research status}

By the end of the nineteenth Century, radio technology began to enter our country. In the early twentieth Century, radio technology in our country has a certain degree of popularity, but did not really develop, until the middle of the twentieth Century, radio technology is developing rapidly. More representative is Beidou Positioning System, it is the positioning system in China's first self-developed, plays a very important role in China's national security and defense construction, and have a huge impact on the terrestrial wireless positioning ${ }^{[14-15]}$. For example, The Hunan University Chen Jie ${ }^{[16]}$ proposed a method for distinguishing the multiple reflection signal false detection signal in complex occlusion environment, the results show that this method can effectively distinguish between LOS, SBP, DBP and the NLOS signal three times or more than three times of reflection.

With the continuous transformation of industry, China's precision requirements for parts and components is getting higher and higher, the demand for size measurement more and more in the automotive, marine, aerospace and hydroelectric power industry , the demand for size measurement more and more, such as aircraft type, car engine overall geometric parameters, The hull shape and synthesis, the blade morphology of large hydro-generators, the geometrical parameters of the particle-accelerated ring, and so on. Therefore, the research and development of the range finder has been paid attention to by researchers ${ }^{[17-18]}$.

In the range finder system, Yang Luna ${ }^{[19]}$ of Northwestern University has developed a low power small pulse laser range finder system. Through the test, the time pulse measurement accuracy reached the picosecond (ps) level, the relative error reached zero percent, but this is just a preliminary exploration, need further study. Zhang Lihong et al. ${ }^{[20]}$ provided a MEGA8 microcontroller as the core of low-cost ultrasonic rangefinder design, the measurement accuracy of up to $0.5 \mathrm{~cm}$, the measurement range can reach $2.5 \mathrm{~m}$. Sun Ting ${ }^{[21]}$ designed a high-precision digital phase measurement algorithm based on full phase spectrum analysis handheld laser range finder, the results show that the rangefinder measuring range of $0.2-50 \mathrm{~m}$, the indication error of about $3 \mathrm{~mm}$.

Comprehensive analysis of domestic and foreign related literature found that the industrial microwave range finder reported less, and the researchers study the purpose is to the low cost of the environment to get accurate ranging positioning. It is well known that the microwave has the characteristics of high precision, strong anti-interference ability and all-weather, and the production cost of microwave range finder is relatively low. Therefore, the research of industrial microwave range finder has the significance of improving the accuracy of distance and reducing the cost and has a huge industrial market demand.

\subsection{Existing problems}

According to the current status of research at domestic and foreign, radio positioning is generally measured by the signal intensity, signal angle, signal propagation delay and other characteristics to measure the distance. Table 2 lists the advantages and disadvantages of the various techniques. 
Table 2 The advantages and disadvantages of various techniques

\begin{tabular}{|c|c|c|}
\hline Signal characteristics & Advantages & Disadvantages \\
\hline Signal strength & $\begin{array}{c}\text { Lower } \\
\text { equipment cost }\end{array}$ & $\begin{array}{l}\text { The noise interference is more sensitive; the non line } \\
\text { of sight signal, the mobility of the device and the } \\
\text { temporal and spatial fluctuation of the channel will } \\
\text { also have a great influence on the position estimation. }\end{array}$ \\
\hline Signal angle & $\begin{array}{c}\text { Low } \\
\text { communication } \\
\text { cost and high } \\
\text { positioning } \\
\text { accuracy }\end{array}$ & $\begin{array}{c}\text { Multipath transmission, NLOS signal and the } \\
\text { accuracy of the angle estimation device are very } \\
\text { sensitive. Not suitable for direct application to } \\
\text { complex nlos. }\end{array}$ \\
\hline $\begin{array}{c}\text { Signal propagation } \\
\text { delay }\end{array}$ & $\begin{array}{l}\text { Get high } \\
\text { positioning } \\
\text { accuracy }\end{array}$ & $\begin{array}{l}\text { Requires time synchronization between the source } \\
\text { and the node to be measured; Sensitive to NLOS } \\
\text { propagation, When the path of sight distance cannot } \\
\text { be detected, it will cause a large positioning error. }\end{array}$ \\
\hline
\end{tabular}

To sum up, improve the ranging is one of the effective ways to improve the positioning accuracy, the traditional range regardless of its signal characteristic value, ranging based on generally is the use of radio wave time difference. Due to the interference of electromagnetic wave, diffraction, the spread of media and obstacles, therefore, the time difference of radio waves will produce inevitable errors. On the other hand, the wavelength of the electromagnetic wave is only related to the frequency, and the wavelength will not change through the interference diffraction. Therefore, it is necessary to conduct in-depth study.

\section{New development trend of wireless positioning and ranging}

Wireless location is an important part of radio technology, and it is an important means to solve wireless positioning technology. With the popularization of open source technology, especially the continuous innovation of computer technology. The development of measuring instruments, towards intelligent, integrated, networked, personalized. In modern wireless ranging technology, the development trend is mainly reflected in the following aspects.

\subsection{Development of new frequency bands}

In recent years, the change of optical communication has become one of the most important parts of the prediction of scientists. From the electromagnetic spectrum can be seen, there is a region has not been developed, microwave and infrared wavelengths between the wavelength of 10-4 10-3m of the gap, the so-called terahertz (THz) ${ }^{[22 ~ 23]}$ Terahertz wave contains the frequency of $0.1 \sim 10 \mathrm{THz}$ (wavelength $0.03 \sim 3 \mathrm{~mm}$ ) electromagnetic wave $(1 \mathrm{THz}=1012 \mathrm{~Hz}$ ), the band is a special area from electronics to photonics transition of the electromagnetic spectrum, and the classical theory of micro transition zone micro quantum theory. The quantum energy of terahertz ( $1 \mathrm{THz}$ is only equivalent to $4 \mathrm{meV}$ ), the signal to noise ratio is very high (more than 1010 ), the frequency is very wide, the landmark is the $0.225 \mathrm{THz}$ airborne radar. If the frequency band is used, it will solve the interference of wireless signal and improve the stability of the wireless location. 


\subsection{Intelligent measuring instrument}

Modern wireless testing has achieved a close combination of measurement and computer. Automatic testing, computer processing and analysis of the instrument has been popular, the level of combination about measurement and computer marks the level of the test system. Digitalization is the development trend of all test instruments. Measurement results and default values can be displayed and stored in digital form, the measurement data can be sent to the external computer by the internal computer, and the automatic test system consisting of standard interface or bus control. The most important point of intelligent instrument is the automation of the testing system, the establishment of automatic test system with intelligent function, not only can improve the test quality, efficiency and accuracy, but also can effectively save resources.

\subsection{Software network measurement}

It is an inevitable trend to expand the network and database of microwave testing instruments. From the development of PC data bus and communication interface standards, the operation of the product, networking faster and faster, more information and more integrated. In the near future, users will be able to achieve a variety of microwave remote sensing devices in different places and remote, personalized, universal, confidential measurement. From the software point of view, Computer embedded real-time control system, through the TCP/IP protocol package provides a variety of measurement technology, so that the computer network measurement platform continues to develop, will bring revolutionary changes. At the same time, the great benefits brought by the network measurement will promote the application of the network measurement in the measurement automation.

\section{Conclusion}

Measurement issues of the Industrial wireless positioning range become more prominent and complex, because its positioning accuracy demanding strict. Arises at the historic moment of the different orientation of strengthening optimization methods have been widely concerned in the field of wireless positioning technology at home and abroad. and has conducted the research. At present, wireless positioning technology has been studied in the new frequency band, intelligent measuring instrument, software network measurement and so on, If we can make a breakthrough in these areas, and effectively applied in practice, will make a significant contribution to the development of human society.

\section{References}

[1] Lu Juntao, Cheng Guoqiang. (2014)Analysis of the development and innovation of radio communication technology. Electronic Technology \&Software Engineering. 2,58.

[2] Huang Qinming. (2007) Research on wireless location algorithm. Shandong University.

[3] Qu Xinghua, Dai Jianfang, Zhang Fumin. (2009)Application study on laser ranging in large-scale measurement. Chinese Journal of Scientific Instrument. 30(3), 481-485.

[4] Shi De-gang, Liu Ye, Wang Feng et al.. (2002)Research of the Ultrasonic Distance-Measure Instrument. Compurter Measurement \& Control. 10(7), 480-482.

[5] Bing Shi, Bing Li. (2003) Principle and application of infrared distance measuring instrument. Domestic IR \& Optoelectronics. 3, 48-49.

[6] Cheng Xianming, Li Shizhong, Qiao Jinjin et al.. (2010) Design and implementation of microwave ranging 
scheme. Sichuan ordnance Journal. 7(31), 96-109.

[7] Wang Xiaopin, Luo Jun, Shen Changxiang. (2012) Study on the stability of three side measurement. Computer engineering and Science. 34(6), 12-17.

[8] Zhong Lihong, Hu Chenquan, Jin Jingji. (2014) Analysis and practice of RSSI based maximum likelihood estimation localization algorithm . Journal of Jilin University. 52(3), 556-560.

[9] Qin Youhua. (2013) Application and development of radio. Research on Urban Construction Theory. 18.

[10]M McGuire Robust (2000) Estimation of Mobile Terminal Position.

[11]Chintalapudi K, Iyer A P, Padmanabhan V N. (2010) Indoor Localization Without the Pain, Proceedings of MobiCom. Chicago Illinois. 173-184.

[12]D H Shin. (2001)Analysis of Positioning Errors in Radio navigation Systems.

[13]Forde L C, Howick E F.(2001) Development of a calibration system for electronic distance measuring instruments. SPIE. 4401, 99-106.

[14]Yi Zhou. (2005) China's GPS Beidou navigation and positioning system. China water transport. 1,45.

[15]Sui Yi. (2004) Research on Beidou satellite and pseudo satellite integrated positioning system. Northwestern Polytechnical University.

[16]Cheng Jie. (2015) Research on the method and technology of 3D wireless location in complex environment. Hunan University.

[17]Kenta Umetsul, Ryosyu Furutnanil, Sanko Osawa, et al..(2005)Geometric calibration of a coordinate measuring machine using a laser tracking system. measurement science and technology 16, 2466-2472.

[18]Li Zongchun, Li Guangyun, Tang Tingsong. et al.. (2005) Application of electronic theodolite intersection measurement system in precision measurement of large antenna. Marine surveying. 25(1), 26-30.

[19]Yang Luna, (2009) Research on low power small pulse laser range finder. Northwestern University.

[20]Zhang Lihong ,Cheng Boju. (2010) Design of a low cost ultrasonic distance measuring instrument. Chemical automation and instrumentation. 37(8), 49-52.

[21]Sun Tin. (2012) Research on handheld laser range finder [D]. Beijing Jiaotong University.

[22]Yao Jianshuan. (2010) Terahertz technology and its application. Journal of Chongqing University of Posts and Telecommunications. (6), 703-707.

[23]Cai He, Guo Xueqiao, He Tin, et al.. (2010) Terahertz technology and application Research Progress. Chinese Optics and Applied Optics. (3), 209-222 